\title{
Microplastics pollution in the Pantanal
}

By now, we all recognise
microplastics as a huge
problem in our oceans. This
awareness peaked when
the threat became direct
to humans, through the
consumption of contaminated
marine produce. Unfortunately,
the situation is even bleaker
than we realised. Dr Pierre
Girard from the Federal
University of Mato Grosso has
discovered microplastics in
the remote Brazilian Pantanal,
a huge and diverse area of
swampland in South America.
This not only threatens an
important breadth of flora and
fauna, but demonstrates the
sheer ubiquity of microplastics,
and the mounting challenge
we face to eradicate them.

The global plastic problem is serious. As we continue to chur out vast quantities of plastic in virtually everything we make, the environment around us is becoming damaged in ways we would never have previously imagined. We're all fish, injured seals, and bottle-ridded fish, injured seals, and botle-ridden new threat has emerged one thats a be a lot more serious - microplastics.

MicrOPLASTICS: WHAT WE KNOW If you're reading this, it's quite likely you've already heard of microplastics. The term refers to any plastic particle smaller than $5 \mathrm{~mm}$ in length, and they can be found in various forms. They are generally a result of the degradation of larger plastics, althoug occasionally (as with microbeads) they are manufactured to be that size. Microplastics are an emerging threat to ecosystems as a whole. Due to their small size they could easily pass alon the food chain and even end up in ecosystems can cause hazardous effects on ecological health, and recently a huge amount of awareness has been raised concerning ocean microplastics. But what about riverine systems, wetlands, or even terrestrial areas?

Unsurprisingly, much of the ocean microplastics originate from rivers
inland. Historically, settlements
are based around rivers inland. Historically, settlements and consequently the bulk of plastic waste originates where urban
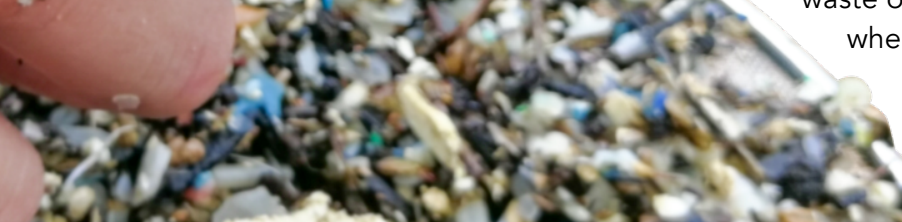

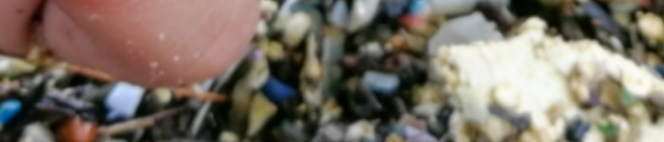

meets water. Not only does this mean that rivers downstream of cities are also highly contaminated with microplastics, but so is any downstream water system they lead to, 作 the Federal University or Mato Grosso presence, of mil, is investigating the American Pantanal.

\section{PROTECTING THE PANTANAL}

The Pantanal is a vast and diverse area Brazil along the border of Paraguay and Bolivia, and floods annually. It supports a huge amount of biodiversity and important ecosystem services. Sadly, despite recognition and protection, the Pantanal is still threatened by anthropogenic disturbance including hydroelectricity, agroindustry, mining and waste dumping. Now microplastics 政

One of the main contributors to the Pantanal is the Cuiabá river. Its tributaries flow through the cities of source of plastic pollution. These cities house up to one million inhabitants and only treat approximately $50 \%$ of their water. The Cuiabá river then flows through a few smaller settlements. before reaching the Pantanal, where flooding peaks in March to April.

In order to present microplastics as a significant threat to the Pantanal, and thus the services and wild life it supports, Dr Girard quantified and classified the microplastic presence in the area. The team decided to sample in both the urban tributaries They used a plankton net (one with

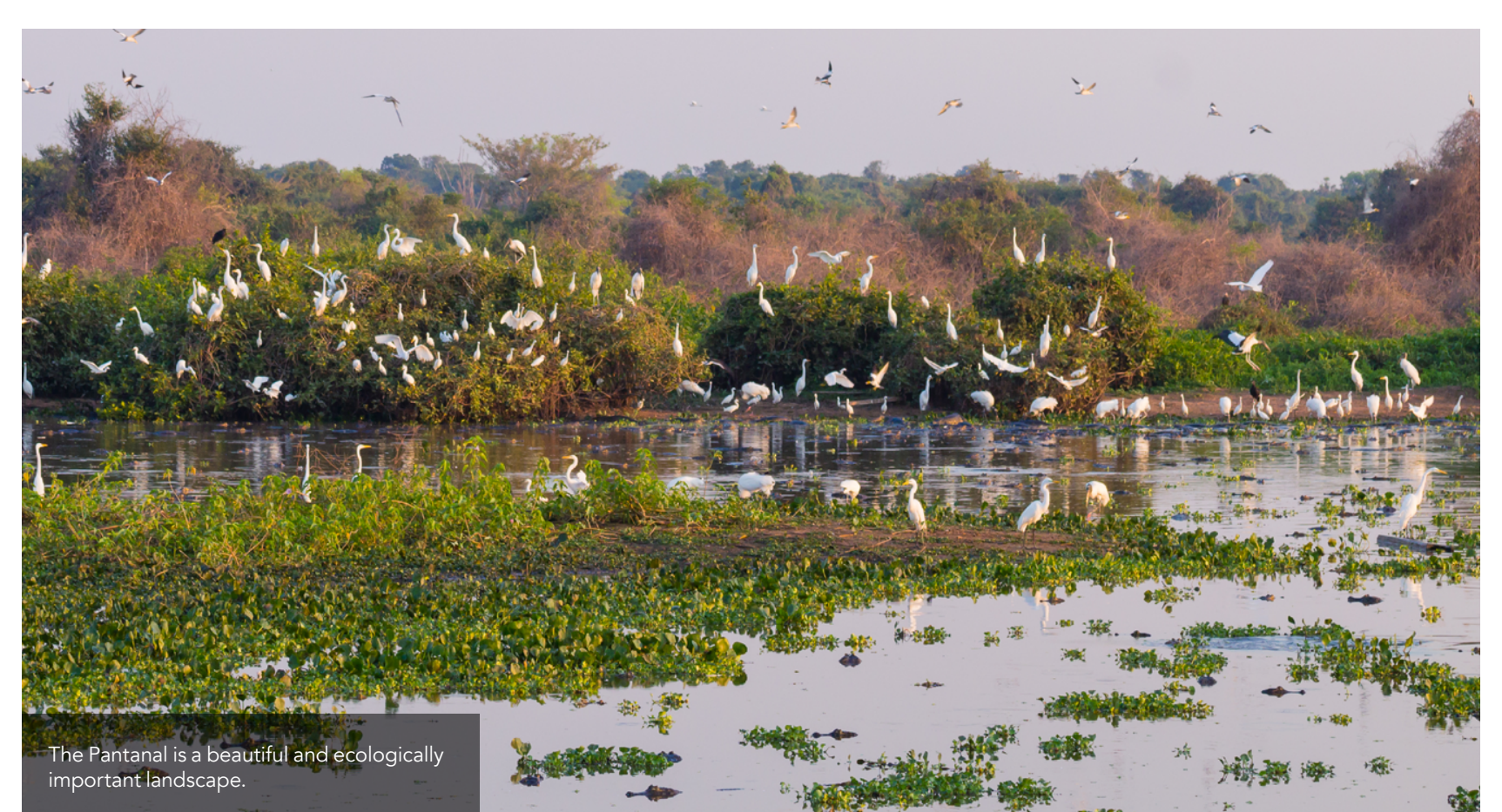

a $68 \mu \mathrm{m}$ mesh size), a method that capture more microplastics than the commonly used neuston net. Samples were filtered back in the lab, examined under a microscope, and categorised. something as small as microplastics, the team were meticulous to minimise the presence of plastic items during this process. They even quantified the atmospheric microplastic levels in the lab, which was concluded to be very low.

\section{ALL SHAPES AND SIZES}

The concentrations were quantified as pean 8 coces pertion lres of water). The average the range of other riverine recordings across the world (although this is a large range). As expected, the concentration in Cuiabá was four times higher than in the Pantanal with an average of 19.9. Again this increase in concentration proximal to urbanisation was mirrored in similar studies across the globe.

Another important measurement was the type and size of the microplastics. Half of all particles were fibres, the rest being fragments (1\%), spherical pellets (22\%) and closed-cell extruded polystyrene particles did not vany much between particles did not vary much between the average size being $192 \mu \mathrm{m}$.
Despite recognition and protection, the Pantanal is still threatened by anthropogenic disturbance... Now microplastics are adding to this.

Fibres are evidently the most serious issue Although these are higher in urbanised in this region. They mostly originate from areas, fibres are the most ubiquitous synthetic clothing and fishing gear, which form of microplastic and will be an mortant research area in the future.

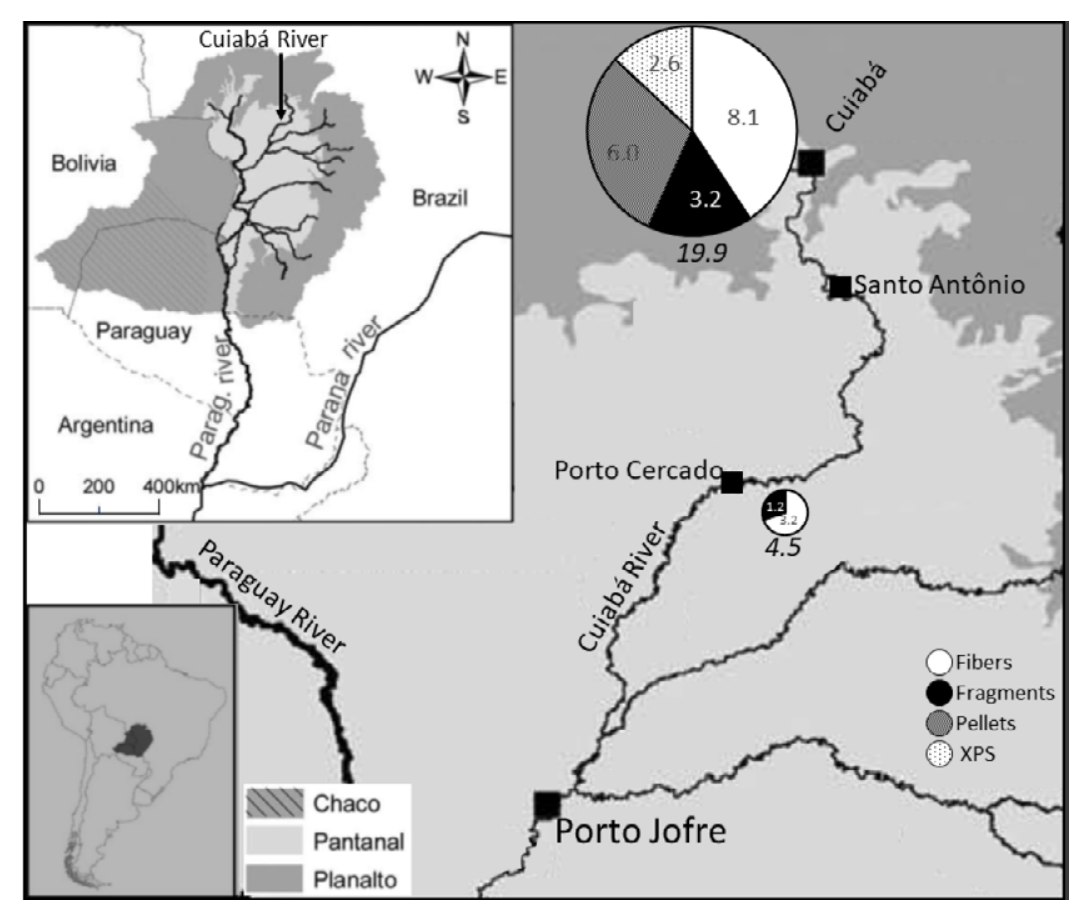

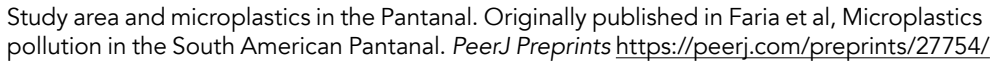
are degraded during use and washing. 
There were virtually no large

beaty products), which suggested that most of the pellets came from

industrial sources instead Interestingly

in the Pantanal, there were 60 times

fewer pellets. This is likely due to

the lack of large industry, and also

gives an idea of the plastic transport

capabilities of the river. Although

fibres also showed a decrease, the

amount was only 2.6 times lower -

likely due to the presence of local

fishing or washing depositing fibres

in the Pantanal floodplain, rather than

increased transport.

The decrease in microplastic

concentration away from urban areas could be due to particles becoming may also be deposited on after periods of flooding drought

or decreased water flow velocity. In

fact, several studies have recorded

the presence of microplastics on

riverbanks and dry floodplains in Brazi and Switzerland. It's also understood that certain shapes and sizes of particle are more susceptible to transport further research in this area might help us effectively target particular plastics.

\section{PREVENTING PLASTIC POLLUTION} It is clear that microplastics have managed to permeate almost all aquatic systems on the globe. Despite a low the plastic solon moving away from the plastic source, transportation continue uncs the plastic prober is addressed. Dr Girard hopes to expand his research and sampling across the Pantanal, covering more land types and even terrestrial areas.

Preliminary studies have also looked at uptake of microplastics in bivalves, a group of filter feeders at the base of the food web. Research has shown that not only do these animals uptake microplastics, but they deposit it on the aquatic substrate (the material on the riverbed, e.g., soll or gravel) during egestion. This likely leads to contamination of bentic or bottom teeders, and also predators of the bivalves themselves. This reinfores one of the
Microplastic sizes in the Cuiabá River tributaries and in the Pantanal

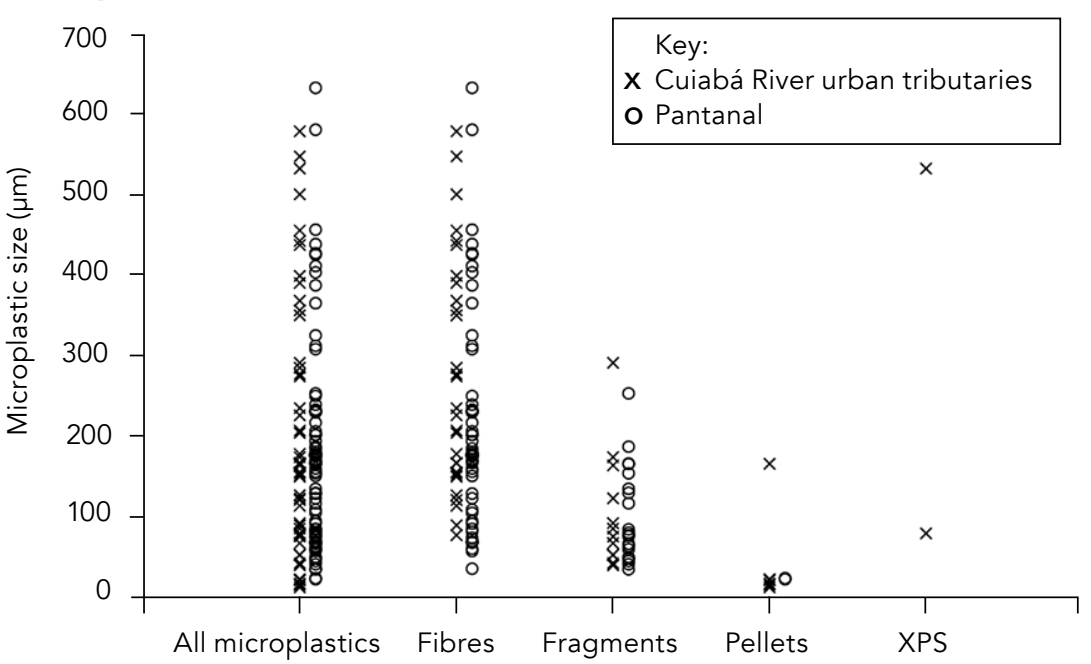

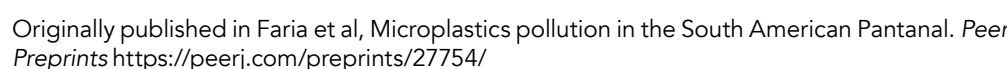

This reinforces one of the most dangerous aspect of microplastics: their ability to readily traverse the food web - a question that is still hotly debated in the scientific literature.

their ability to readily traverse the food a strong case can be put forward web-a question that is still hotly debated to policy makers looking to make in the scientific literature.

After expanding his research study area and sampling sites, Dr Girard will start to look closely at the relationship and uptake of microplastics in aquatic organisms. Provided there is suitable and sufficient evidence for both a Microplastics risk not only decreasing the water quality of this pristine and unique habitat but contaminating much of its biodiversity. Such is the reliance of human livellyod and ecosystem services on the Pantanal, that this is also be rapidly addressed.

Microplastic composition
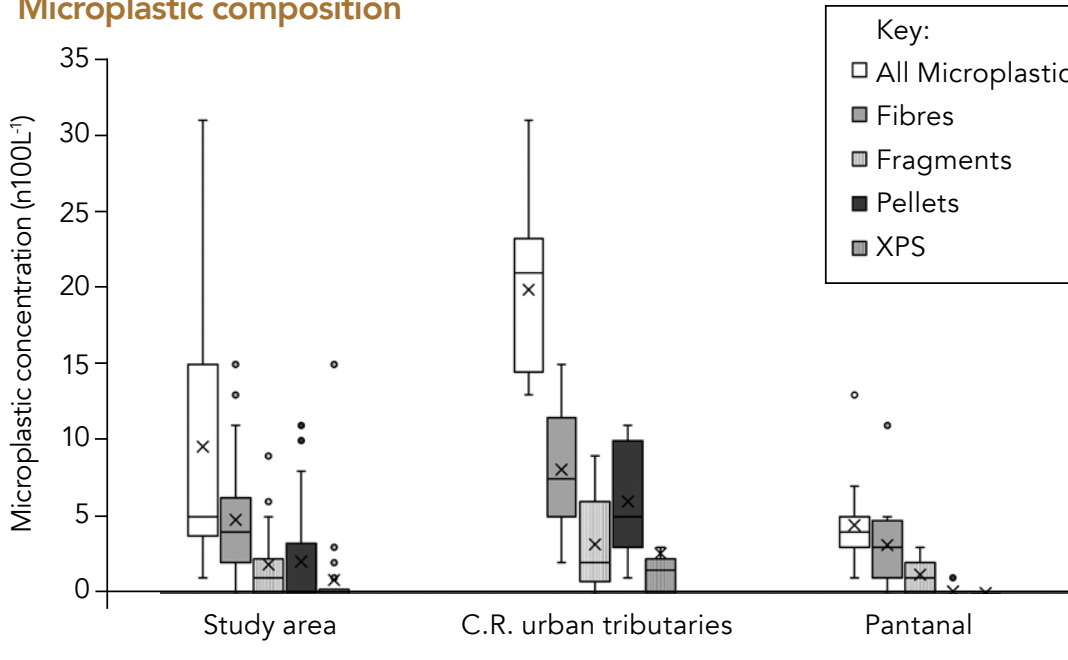

Originally published in Faria et al, Microplastics pollution in the South American Pantanal. Peers

\section{Dehind the Research}

\section{Dr Pierre Girard}

E: pierregirard1301@gmail.com T: +5565993634636

Research Objectives

Dr Pierre Girard's work examines the prevalence of microplastics in the unique Pantanal swampland of South America.

\section{Detail}

Pierre Girard

Av. Fendade Federal de Mato Grosso - UFMT

da Costa, $n^{\circ} 2367$

Bairro Boa Esperança

Brazil

Bio

Pierre Girard is a Canadian living in Brazil. He teaches at dedicated his research to the Pantanal. He has published papers on the Pantanal water dynamics including pollution in the Pantanal wetlands.

\section{Collaborators
- Erika Faria}

- Erika Faria Moreschi

- Carolina Nardes

- Monica Costa

- Susete W. Christo

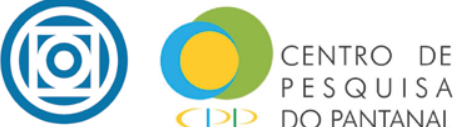

UFMT pantana

\section{References}

Faria E., Girard P., Nardes C. S., Moreschi A., Christo S. W. Ferreira Junior A. L., Costa M. F. 2019. Microplastics pollution https//doiorg/10.7287/peri. Pepin 27754 i

\section{Personal Response}

\section{How long is it expected to take before an}

\section{we}

II Reducing plastic use is "the" answer to plastic pollution everywhere in the world. In the Pantanal area this is unlikely to occur in the next decade, if left to governments and industry initiative. Sewage treatmen researched, but even if they were to become available unlikely to be resolved in Brazil and the Pantanal anywhere soon. Activism to raise public awareness plastic production and use is the most repid form of intervention.

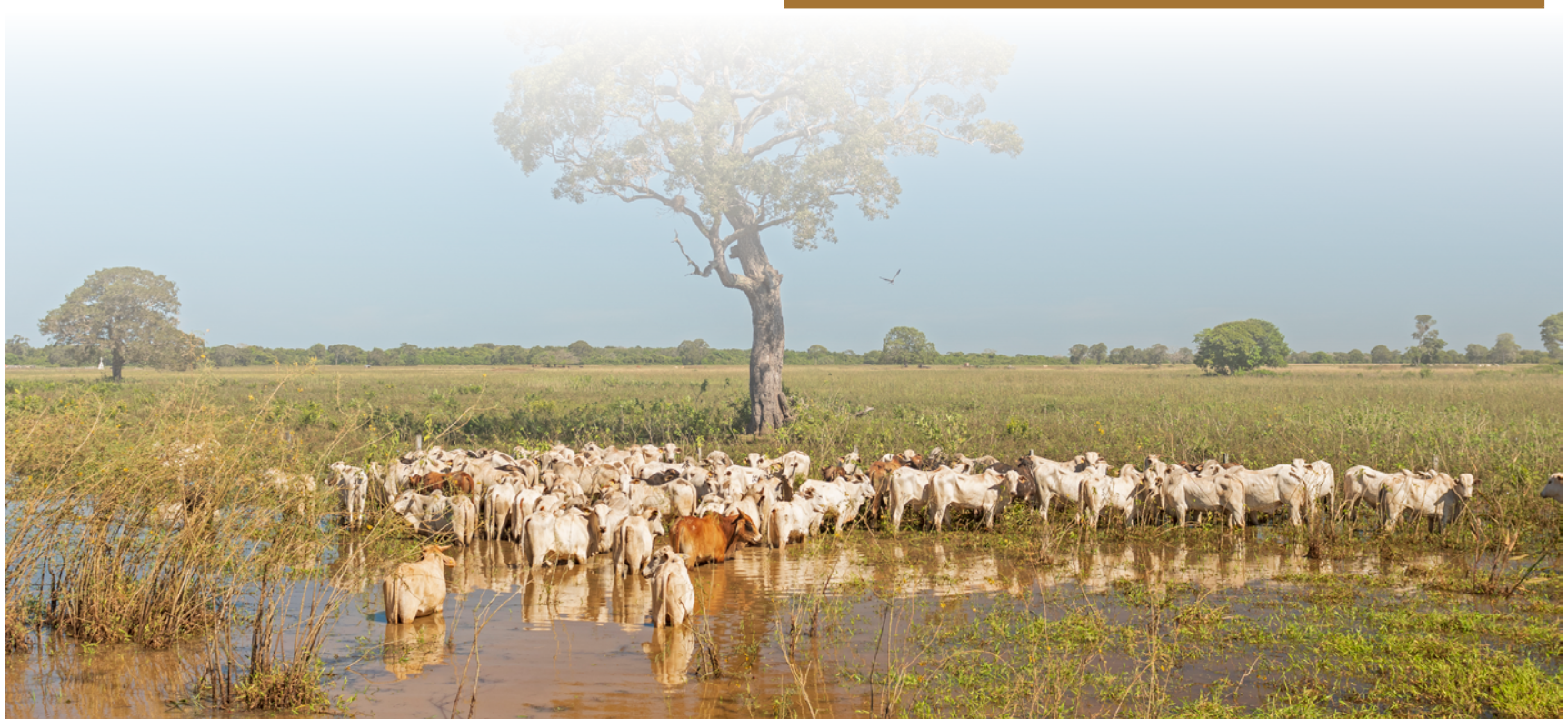

